\title{
Naked mole-rat cortical neurons are resistant to acid-induced cell death
}

\author{
Zoé Husson and Ewan St. John Smith ${ }^{*}$
}

\begin{abstract}
Regulation of brain $\mathrm{pH}$ is a critical homeostatic process and changes in brain $\mathrm{pH}$ modulate various ion channels and receptors and thus neuronal excitability. Tissue acidosis, resulting from hypoxia or hypercapnia, can activate various proteins and ion channels, among which acid-sensing ion channels (ASICs) a family of primarily $\mathrm{Na}^{+}$permeable ion channels, which alongside classical excitotoxicity causes neuronal death. Naked mole-rats (NMRs, Heterocephalus glaber) are long-lived, fossorial, eusocial rodents that display remarkable behavioral/cellular hypoxia and hypercapnia resistance. In the central nervous system, ASIC subunit expression is similar between mouse and NMR with the exception of much lower expression of ASIC4 throughout the NMR brain. However, ASIC function and neuronal sensitivity to sustained acidosis has not been examined in the NMR brain. Here, we show with whole-cell patch-clamp electrophysiology of cultured NMR and mouse cortical and hippocampal neurons that NMR neurons have smaller voltage-gated $\mathrm{Na}^{+}$channel currents and more hyperpolarized resting membrane potentials. We further demonstrate that acid-mediated currents in NMR neurons are of smaller magnitude than in mouse, and that all currents in both species are reversibly blocked by the ASIC antagonist benzamil. We further demonstrate that NMR neurons show greater resistance to acid-induced cell death than mouse neurons. In summary, NMR neurons show significant cellular resistance to acidotoxicity compared to mouse neurons, contributing factors likely to be smaller ASIC-mediated currents and reduced NaV activity.
\end{abstract}

Keywords: ASIC, Acid-induced currents, Acidotoxicity, Naked mole-rat, Hippocampus

\section{Introduction}

Acid-sensing channels (ASICs) are ion channels of the $\mathrm{ENaC} /$ Deg superfamily and most subunits are activated by extracellular protons $[1,2]$. Six different ASIC subunits are encoded by 4 ASIC genes (ASIC1a, ASIC1b, ASIC2a, ASIC2b, ASIC3 and ASIC4), which assemble as homo- or heterotrimers [3]; neither ASIC2b nor ASIC4 form protonsensitive homotrimers. ASICs are primarily permeable to $\mathrm{Na}^{+}$, although ASICla homomeric channels are also $\mathrm{Ca}^{2+}$ permeable [2]. In the central nervous system (CNS), neurons have been shown to primarily express ASICla homomers and heteromers of ASICla/2a and ASICla/2b [4-8], where they have been demonstrated to have key roles in synaptic plasticity [9-11] and fear conditioning [12-14], as well as being major players in neuronal death resulting from brain ischemia [5, 15-17], and neurodegenerative diseases [18-20].

\footnotetext{
* Correspondence: es336@cam.ac.uk

Department of Pharmacology, University of Cambridge, Tennis Court Road, Cambridge CB2 1PD, UK
}

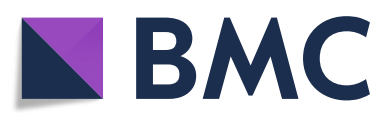

(c) The Author(s). 2018 Open Access This article is distributed under the terms of the Creative Commons Attribution 4.0 International License (http://creativecommons.org/licenses/by/4.0/), which permits unrestricted use, distribution, and

reproduction in any medium, provided you give appropriate credit to the original author(s) and the source, provide a link to the Creative Commons license, and indicate if changes were made. The Creative Commons Public Domain Dedication waiver (http://creativecommons.org/publicdomain/zero/1.0/) applies to the data made available in this article, unless otherwise stated. portant process [21]. Brain tissue acidosis can result either from an increase in tissue partial pressure of carbon dioxide $\left(\mathrm{PCO}_{2}\right)$ during hypercapnia, or from the accumulation of the byproducts of anaerobic metabolism, such as lactate and protons, during hypoxia [22]. During periods of tissue acidosis, activation of ASICs by extracellular acidification is worsened by the release of allosteric modulators such as lactate [23], spermine [16] and arachidonic acid [24, 25]. In addition to the activation of the $\mathrm{Ca}^{2+}$ permeable ASIC1a channel $[15,16]$, a drop in $\mathrm{pH}$ also modulates the activity of numerous others ion channels, including voltage-gated ion channels [26-29] and glutamate receptors [30,31], therefore leading to disturbance in ion homeostasis, excitotoxicity and ultimately neuronal death $[5,15,16]$.

Naked mole-rats (NMRs, Heterocephalus glaber) are subterranean rodents belonging to the Bathyergidae African mole-rat family found in East Africa [32]. Unusually for a mammal, NMRs are eusocial $[33,34]$. However, NMRs also 
display a range of remarkable physiological peculiarities, which is beginning to make a significant impact on biomedical research [35]. The unusual physiology of the NMR includes: extreme longevity with no increased risk of death with ageing [36], an apparent absence of age-related neurodegenerative disorders [37, 38], resistance to cancer [39-41], insensitivity to certain noxious and irritant stimuli [42-45] and hypoxia/hypercapnia resistance resulting from altered NMDA receptor function and an ability to utilize fructose as an energy source [46-49]. It is striking that NMRs are resistant to many pathological conditions known to involve ASICs. Recordings of ASIC-mediated currents in dorsal root ganglion (DRG) sensory neurons demonstrated an increased frequency and magnitude of ASIC responses in NMR neurons compared to mouse neurons [43], with APETx2, an inhibitor of ASIC3-containing ASICs, demonstrating a key role for ASIC3, even though nmrASIC3 does not appear to form functional homotrimers [50]. Previously we mapped out ASIC expression in different NMR brain regions and observed similar expression between mouse and NMR, a key exception being much lower ASIC4 levels throughout the NMR brain [51], however, no one has yet studied the function of ASICs in NMR brain neurons.

In this study, we investigated acid-induced currents in mouse and NMR neurons using whole-cell patch clamp recording of cultured neonatal hippocampal and cortical neurons. We find that NMR neurons have ASIC-mediated currents of significantly smaller peak current amplitude than those recorded from mouse neurons and that NMR neurons are resistant to acid-induced cell death. Overall, these results suggest that the reduced acid-induced cell death in NMR neurons may be neuroprotective.

\section{Methods}

\section{Animals}

All experiments were conducted in accordance with the United Kingdom Animal (Scientific Procedures) Act 1986 Amendment Regulations 2012 under a Project License (70/7705) granted to E. St. J. S. by the Home Office; the University of Cambridge Animal Welfare Ethical Review Body also approved procedures. Breeding couples of 1 male and 2 female C57/bl6 mice were conventionally housed with nesting material and a red plastic shelter; the holding room was temperature-controlled $\left(2{ }^{\circ} \mathrm{C}\right)$ and mice were on a normal 12-h light/dark cycle with food and water available ad libitum. Naked mole-rats were bred in house and maintained in a custom-made caging system with conventional mouse/rat cages connected by different lengths of tunnel. Bedding and nesting material were provided along with running wheels and chew blocks. The room was warmed to $28{ }^{\circ} \mathrm{C}$ and humidified, with a heat cable to provide extra warmth running under $2-3$ cages, and red lighting (08: 00-16:00) was used.

\section{Neuronal cultures}

P0-P2 mice and P0-P5 naked mole-rats were used to prepare cortical and hippocampal neuronal cultures. Multiple pups (2-4) were used to prepare a single culture. Following decapitation, heads were immediately placed in dishes containing ice-cold Hank's Balanced Salt Solution (HBSS) solution $(20 \mathrm{mM}$ HEPES, $30 \mathrm{mM}$ glucose in HBSS, Life Technologies). Brains were removed, transferred to a new dish and the two hippocampi and cortices were isolated. Tissues were subsequently incubated in an enzymatic digestion solution: $2 \mathrm{mg} / \mathrm{mL}$ papain (Worthington Biochemical Corporation) in Hibernate- $\mathrm{Ca}^{2+}$ solution (Brain Bits), activated by $0.5 \mathrm{mM}$ Glutamax (Life Technologies) at $37^{\circ} \mathrm{C}$ for $30 \mathrm{~min}$ in a $5 \% \mathrm{CO}_{2}$ incubator. The digestion solution was then replaced by HBSS solution supplemented with DNAse I (250 Kunitz units/mL, Sigma Aldrich) and tissues were slowly triturated (5-7 times) using a P1000 pipette. Neuronal suspensions were filtered through a $100 \mu \mathrm{m}$ nylon cell strainer (Corning) to remove non-dissociated pieces of tissues before centrifugation for 5 mins at $1100 \mathrm{rpm}$ at room temperature. Supernatants were discarded and the pellets carefully resuspended in HBSS solution. After further centrifugation for $5 \mathrm{~min} 1100 \mathrm{rpm}$ at room temperature, pellets were resuspended in MEM/HS solution: $10 \%$ heat-inactivated horse serum (Life Technologies), $2 \mathrm{mM}$ glucose, $0.0025 \%$ Glutamax, and $0.2 \mathrm{mg} / \mathrm{mL}$ primocin (InVivogen). Hippocampal and cortical neurons were plated on $35 \mathrm{~mm}$ plastic dishes (Fisher Scientific), previously coated with $100 \mathrm{mg} / \mathrm{mL}$ poly-L-lysine (Sigma-Aldrich), rinsed with water and dried, at a density of 300,000 cells $/ \mathrm{mL}(2 \mathrm{~mL} /$ dish $)$. After a 4-h incubation in a $37{ }^{\circ} \mathrm{C} /$ $5 \% \mathrm{CO}_{2}$ incubator, the MEM/HS solution was removed and the dishes were flooded with Neurobasal/B27 solution (1X B27 Supplement, 0.0025\% Glutamax, and $0.2 \mathrm{mg} / \mathrm{mL}$ primocin). Naked mole-rat neurons were kept at $33{ }^{\circ} \mathrm{C}$ in $5 \% \mathrm{CO}_{2}$ incubator, whereas mouse neurons were kept at $37{ }^{\circ} \mathrm{C}$ in a $5 \% \mathrm{CO}_{2}$ incubator; this is due to NMRs being cold-blooded and NMR cells do not withstand $37{ }^{\circ} \mathrm{C}$ for long periods of time [52]. Half of the medium was exchanged for fresh medium every 2-3 days until the cultures were used for experiments.

\section{Electrophysiology}

Hippocampal and cortical neurons from mouse and NMR were used for whole-cell patch-clamp recordings at 9-12 days in vitro (DIV9-12). Recordings were performed at room temperature using the following solutions: extracellular (in $\mathrm{mM}$ ) $-140 \mathrm{NaCl}, 4 \mathrm{KCl}, 2 \mathrm{CaCl}_{2}$, $1 \mathrm{MgCl}_{2}, 4$ glucose, 10 HEPES, adjusted to $\mathrm{pH} 7.4$ with $\mathrm{NaOH}$ and 300-310 mOsm with sucrose; intracellular (in $\mathrm{mM}$ ) - $110 \mathrm{KCl}, 10 \mathrm{NaCl}, 1 \mathrm{MgCl}_{2}, 1$ EGTA, 10 HEPES, $2 \mathrm{Na}_{2}$ ATP, $0.5 \mathrm{Na}_{2} \mathrm{GTP}$, adjusted to $\mathrm{pH} 7.3$ with $\mathrm{KOH}$ and to $310-315 \mathrm{mOsm}$ with sucrose. Acidic extracellular solutions were made using MES ( $\mathrm{pH}$ 5.0). Patch 
pipettes were pulled (Model P-97, Flaming/Brown puller; Sutter Instruments,) from borosilicate glass capillaries (Hilgenberg $\mathrm{GmbH}$ ) and had a resistance of 6-10 $\mathrm{M} \Omega$. Data were acquired using an EPC10 amplifier and Patchmaster software (HEKA). Whole-cell currents were recorded at $20 \mathrm{kHz}$, pipette and membrane capacitance were compensated using Patchmaster macros, and series resistance was compensated by $>60 \%$. Cell capacitances and resting membrane potentials were measured just after cell opening in whole-cell configuration. To study macroscopic voltage-gated currents, a standard voltagestep protocol was used whereby cells were held at $120 \mathrm{mV}$ for 200 msecs before stepping to the test potential $(-80 \mathrm{mV}-+65 \mathrm{mV}$ in $5 \mathrm{mV}$ increments) for 50 msecs, returning to the holding potential $(-60 \mathrm{mV})$ for 200 msecs between sweeps. In some experiments, tetrodotoxin (300 nM, Alomone Labs) was perfused for $30 \mathrm{~s}$ before repeating the voltage-step protocol. To measure neuronal acid-sensitivity, cells were exposed to the following protocol: $5 \mathrm{~s}$ of $\mathrm{pH} 7.4$; 5 -s of $\mathrm{pH} 5$; and $5 \mathrm{~s}$ of $\mathrm{pH}$ 7.4. ASIC antagonists (100 $\mu \mathrm{M}$ Benzamil, Sigma) were perfused during $30 \mathrm{~s}$ before applying another $5 \mathrm{~s}$ pulse of $\mathrm{pH} 5$. After $90 \mathrm{~s}$ wash time with $\mathrm{pH} 7.4$ solution, a $5 \mathrm{~s}$ pulse of $\mathrm{pH} 5$ was applied to check for reversal of any block observed. Current amplitude was measured in Fitmaster (HEKA) by taking the maximum peak response and subtracting the mean baseline amplitude in the preceding $50 \mathrm{msec}$ (voltage-gated currents) or $\sim 2.5 \mathrm{~s}$ (ASIC currents); current amplitude was normalized for cell size by dividing by cell capacitance. Using Igor Pro, for each individual cell that underwent the voltage-step protocol, the following equation was fitted to the normalized inward currents:

$$
i(x)=\Gamma \cdot x \cdot \frac{1-e^{-\frac{x-\text { Erev }}{25 m V}}}{1-e^{-\frac{x}{25 m V}}} \cdot \frac{1}{\left(1-e^{-\frac{x-V H a l f}{\text { slope }}}\right)^{3}}
$$

where Erev is the reversal potential; Vhalf the halfactivating potential; $\Gamma$ a constant and $\mathrm{x}$ the command potential. Similarly, a Boltzmann equation was fitted to the normalized outward currents:

$$
\frac{I}{\operatorname{Imax}}=\frac{1}{1+e^{\frac{(V \text { Half }-V m)}{\text { slope }}}}
$$

where $\mathrm{Vm}$ is the membrane voltage and VHalf the voltage at half-maximal activation. To determine the inactivation time of the ASIC-mediated currents, a single exponential was fitted. Data are expressed as mean \pm standard error of the mean (SEM). Using Prism (GraphPad), paired t-tests were used to compare the effects of ASIC antagonists on proton-gated currents within both mouse and NMR neuron datasets; unpaired t-tests were used to compare parameters, such as neuronal resting membrane potential and capacitance and ASICmediated current amplitude, between mouse and NMR neuron datasets.

\section{Acid-induced cell death assays}

Mouse and NMR neurons were used to measure acidinduced cell death at DIV9-12. The $\mathrm{pH} 7.4$ and $\mathrm{pH} 5$ extracellular solutions used were the same as those described above for electrophysiology experiments. Neuronal cultures from both mouse and NMR were rinsed twice with $37{ }^{\circ} \mathrm{C}$ solution (pH 7.4 or pH 5) and then incubated with $\mathrm{pH} 7.4$ or $\mathrm{pH} 5$ solution for $2 \mathrm{~h}$ at $37^{\circ} \mathrm{C}$. Cultures from both conditions were then rinsed with warm $\mathrm{pH}$ 7.4 solution and incubated during $30 \mathrm{~min}$ with $\mathrm{pH} 7.4$ solution containing $1.5 \mathrm{mM}$ propidium iodide (PI, Sigma Aldrich) to stain necrotic cells and Hoechst 33,342 (dilution 1/2500, Sigma) to label all nuclei. Labelled cultures were imaged using an epifluorescence microscope (Olympus) equipped with a $20 \mathrm{X}$ objective (Olympus) and a QImaging camera. To determine the percentage of dead necrotic PI-positive cells, we used the software Fiji to count the total number of cells per field of view by counting nuclei on the Hoechst images, and subsequently counting the number of PI-positive nuclei on the PI images. One to three dishes per condition were used, and three different images per dish were taken. Data were collected from three different cultures and each culture was prepared from multiple animals. A one-way ANOVA test (Prism, GraphPad) corrected for multiple comparisons (Tukey test) was used to compare the percentage of cell death in each field of view at pH 7.4 and $\mathrm{pH} 5$ in both species. Data are expressed as mean \pm standard error of the mean (SEM).

\section{Results}

Basic electrophysiological properties and voltage-gated $\mathrm{Na}^{+}$channel activity differ between NMR and mouse neurons

Electrophysiological recordings from NMR neurons have been performed in both DRG sensory neurons $[43,50]$ and CNS neurons $[46,47,53]$. However, neuronal activity from NMR CNS has only been recorded in brain slices, in the form of field excitatory postsynaptic potentials, and the basic electrophysiological properties of NMR neurons in hippocampal and cortical cultures have not yet been described.

We first compared the capacitance and resting membrane potential of NMR and mouse neurons from both cortical and hippocampal neuronal cultures (Fig. 1). The capacitance of NMR neurons was significantly smaller than in mouse neurons in both cortical and hippocampal cultures (cortex: 17.27 $\pm 1.02 \mathrm{pF}$ versus $28.72 \pm 2.32 \mathrm{pF}$ for NMR $(n=30)$ and mouse $(n=24)$ neurons, respectively; hippocampus: $17.41 \pm 1.03 \mathrm{pF}$ versus $38.13 \pm 3$. 


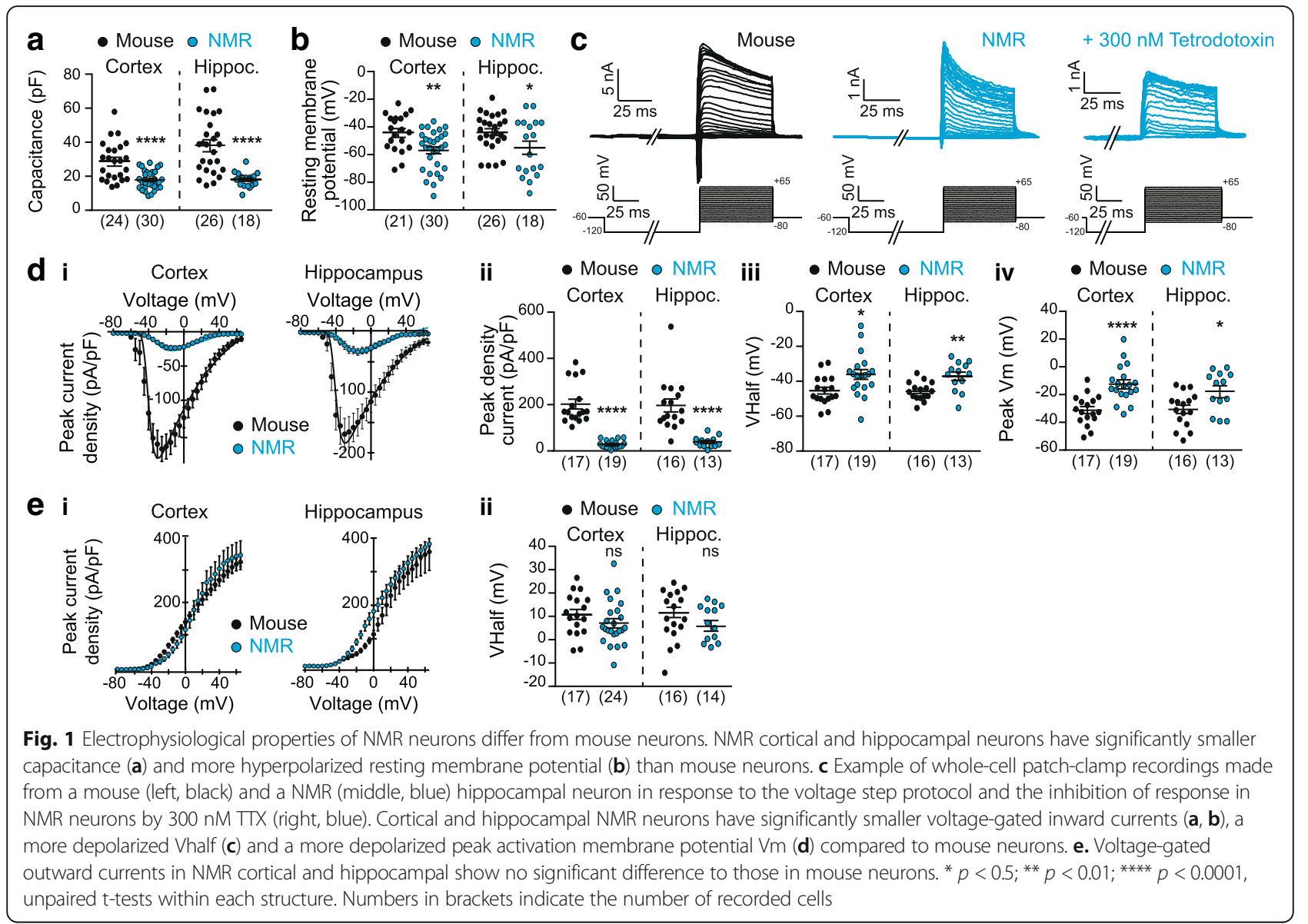

$27 \mathrm{pF}$ for NMR $(n=18)$ and mouse $(n=26)$ neurons, respectively; unpaired two-sided t-tests, $\% * \%<0.0001$, Fig. 1a). Resting membrane potentials were measured as soon as the whole-cell configuration was established and NMR neurons were significantly more hyperpolarized than mouse neurons, in both cortical and hippocampal cultures (cortex: $-57.03 \pm 2.64 \mathrm{mV}$ versus $-44.05 \pm 2$. $84 \mathrm{mV}$ for NMR $(n=30)$ and mouse $(n=21)$ neurons, respectively; hippocampus: $-55.11 \pm 4.79 \mathrm{mV}$ versus $43.85 \pm 2.59 \mathrm{mV}$ for NMR $(n=18)$ and mouse $(n=26)$ neurons, respectively; unpaired two-sided t-tests; $* * 0<$ $0.01 ; * p<0.05$, Fig. $1 \mathrm{~b})$.

We then investigated macroscopic voltage-gated currents in NMR and mouse neurons, using a voltage-step whereby cells were held at $-120 \mathrm{mV}$ for 200 msecs before stepping to the test potential $(-80 \mathrm{mV}$ to $+65 \mathrm{mV}$ in $5 \mathrm{mV}$ increments) for $50 \mathrm{msecs}$, and returning to the holding potential $(-60 \mathrm{mV})$ for 200 msecs between sweeps (Fig. 1c). Both NMR and mouse neurons showed inward and outward currents (Fig. 1c-e). In some experiments, the voltage-step protocol was run twice, the second time after $300 \mathrm{nM}$ tetrodotoxin (TTX) had been applied for $30 \mathrm{~s}$ to investigate the contribution of TTXsensitive voltage-gated $\mathrm{Na}^{+}$channels $(\mathrm{NaVs})$ to the macroscopic voltage-gated inward currents recorded in NMR neurons (Fig. 1c, right panel). In both cortical and hippocampal NMR neurons, the voltage-gated inward currents were fully blocked by $300 \mathrm{nM}$ TTX ( $n=8$ and $n=2$ for cortical and hippocampal neurons, respectively). The fact that no inward current remained after application of 300 nM TTX indicates that only NaVs were activated with our voltage-step protocol and that there was no measurable contribution of voltage-gated $\mathrm{Ca}^{2+}$ channels to the inward currents recorded. Moreover, these results indicate that cortical and hippocampal NMR neurons only express TTX-sensitive NaVs.

Strikingly, NMR neurons had a significantly smaller inward peak current density in both cortical and hippocampal cultures (cortex: $28.79 \pm 3.83 \mathrm{pA} / \mathrm{pF}$ versus 202 . $40 \pm 21.35 \mathrm{pA} / \mathrm{pF}$ for NMR $(n=19)$ and mouse $(n=17)$ neurons, respectively; hippocampus: $39.00 \pm 6.39 \mathrm{pA} / \mathrm{pF}$ versus $196.70 \pm 28.28 \mathrm{pA} / \mathrm{pF}$ for $\mathrm{NMR}(n=13)$ and mouse $(n=16)$ neurons, respectively; unpaired twosided t-tests, $* * * 0.0001$, Fig. 1d.i-ii). Additionally, the voltage of half-activation (Vhalf) and the peak inward current amplitude potential (peak Vm) were more depolarized in NMR neurons compared to mouse neurons (cortex: Vhalf: $-36.12 \pm 2.80 \mathrm{mV}$ versus $-45.42 \pm 1$. 
$99 \mathrm{mV}$ and peak $\mathrm{Vm}:-12.43 \pm 3.02 \mathrm{mV}$ versus $-31.27 \pm$ $2.61 \mathrm{mV}$ for NMR $(n=19)$ and mouse $(n=17)$ neurons, respectively; hippocampus: Vhalf: $-37.10 \pm 2.34 \mathrm{mV}$ versus $-45.71 \pm 1.30 \mathrm{mV}$ and peak $\mathrm{Vm}:-17.62 \pm 4.00 \mathrm{mV}$ versus $-30.68 \pm 3.09 \mathrm{mV}$ for NMR $(n=13)$ and mouse $(n=16)$ neurons, respectively; unpaired two-sided $\mathrm{t}$ tests, ${ }^{* * * * * *} p<0.0001$, ${ }^{* * *} p<0.01 ;{ }^{*} p<0.05$, Fig. 1d.iii-iv). These results suggest that NMR neurons may be less excitable compared to mouse neurons, with more hyperpolarized resting membrane potentials and smaller voltage-gated inward currents that are activated at more depolarized potentials, i.e. a greater depolarizing stimulus is required to activate NMR voltage-gated inward currents that produce much smaller currents.

By contrast, current-voltage curves for voltage-gated outward currents were similar between NMR and mouse neurons from both cortical and hippocampal neurons (Fig. 1e.i) and Vhalfs were not significantly different (cortex: $7.13 \pm 1.91 \mathrm{mV}$ versus $10.77 \pm 2.18 \mathrm{mV}$ for NMR $(n=24)$ and mouse $(n=17)$ neurons, respectively; hippocampus: $5.15 \pm 2.50 \mathrm{mV}$ versus $11.59 \pm 2.26 \mathrm{mV}$ for NMR $(n=14)$ and mouse $(n=16)$ neurons, respectively; unpaired two-sided t-tests, $p=0.221$ and $p=0.094$ for cortex and hippocampus respectively, Fig. 1e.ii).

\section{Acid-induced currents are mediated by ASICs in both NMR and mouse neurons}

The expression profile of ASIC subunits is similar in mouse and NMR brains, with the exception of lower levels of ASIC4 throughout the NMR brain [51], results suggesting that functional ASIC-mediated currents should be present in NMR as others have shown in mouse $[4,5,7,8,15]$.

A $5 \mathrm{~s}$ pulse of $\mathrm{pH} 5$ was applied to NMR and mouse neurons from both hippocampal and cortical neurons and rapidly activating and inactivating acid-induced responses were recorded in every cell of both species (Fig. 2a). However, the peak current density of acid-mediated responses recorded in NMR neurons was significantly smaller than in mouse neurons, in both hippocampal and cortical
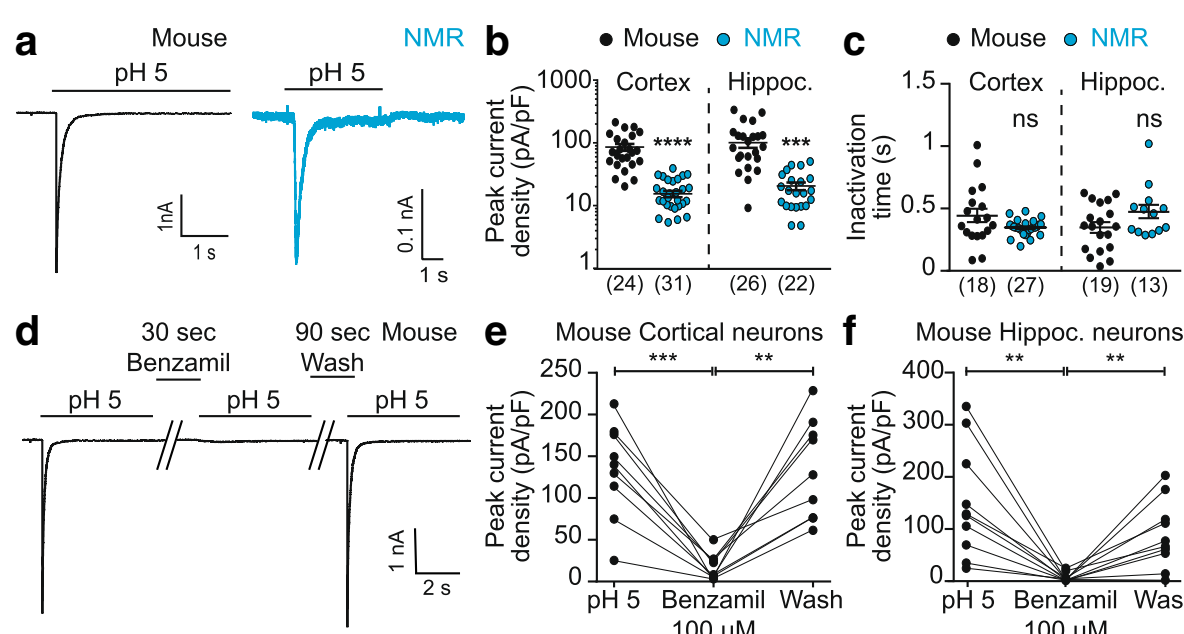

$100 \mu \mathrm{M}$
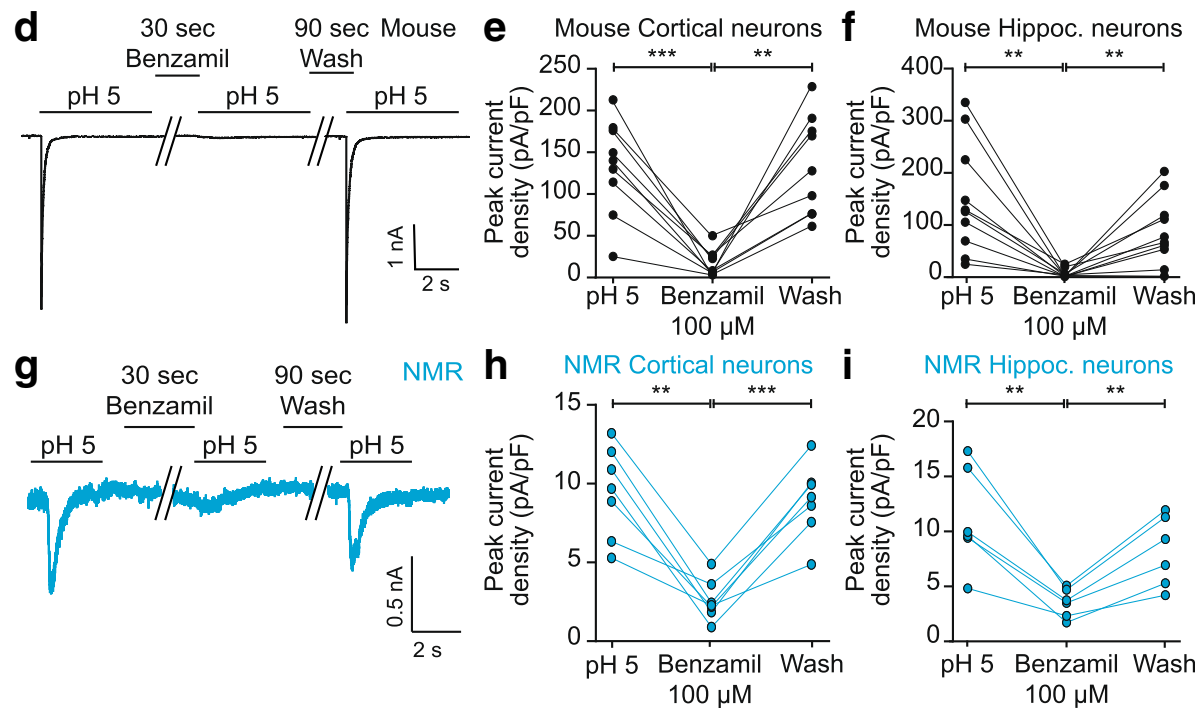

Fig. 2 ASICS mediate acid-induced currents in NMR and mouse CNS neurons. a Both mouse (black trace, left) and NMR (blue trace, right) neurons respond to a pH 5 solution with a transient inward current. b Acid-induced currents are of significantly smaller amplitude in NMR neurons compared to mouse neurons. $\mathbf{c}$ Inactivation time constants of the acid-induced responses are similar between NMR and mouse neurons. $\mathbf{d}$ Example trace of an acid-induced current elicited by a pH 5 solution and the effect of $100 \mu \mathrm{M}$ benzamil in a mouse cortical neuron. e, f Acid-induced currents in both cortical and hippocampal mouse neurons were reversibly blocked by $100 \mu \mathrm{M}$ benzamil ( $n=9$ and $n=10$ cortical and hippocampal neurons, respectively). g Example trace of an acid-induced current evoked by a pH 5 solution in a cortical NMR neuron showing inhibition by $100 \mu \mathrm{M}$ benzamil. $\mathbf{h}, \mathbf{i}$ In both cortical $(n=7)$ and hippocampal $(n=6)$ NMR neurons, acid-induced currents were reversibly blocked by $100 \mu \mathrm{M}$ benzamil. ${ }^{* *} p<0.01$; ${ }^{* * *} p<0.0001$, one-way ANOVA paired tests, Tukey's multiple comparison tests. Numbers into brackets indicates the number of recorded cells 
neurons (cortex: $15.41 \pm 1.82 \mathrm{pA} / \mathrm{pF}$ versus $85.66 \pm 10.90$ $\mathrm{pA} / \mathrm{pF}$ for NMR $(n=31)$ and mouse $(n=24)$ neurons, respectively; hippocampus: $20.54 \pm 2.86 \mathrm{pA} / \mathrm{pF}$ versus 100 . $90 \pm 17.68 \mathrm{pA} / \mathrm{pF}$ for NMR $(n=22)$ and mouse $(n=26)$ neurons, respectively; unpaired two-sided t-tests, ${ }^{* * * * *} p<$ 0.0001 , **: $p<0.001$, Fig. 2b). By contrast, the inactivation time constant of the acid-mediated currents was similar between NMR and mouse neurons (cortex: $0.35 \pm 0.012 \mathrm{~s}$ versus $0.44 \pm 0.056 \mathrm{~s}$ for $\mathrm{NMR}(n=27)$ and mouse $(n=$ 18) neurons, respectively, unpaired two-sided t-test, $p=0$. 0516; hippocampus: $0.47 \pm 0.06$ s versus $0.35 \pm 0.04$ s for NMR $(n=13)$ and mouse $(n=19)$ neurons, respectively; unpaired two-sided t-test, $p=0.0861$, Fig. $2 \mathrm{c}$ ).

The transient nature of the acid-mediated inward currents in both NMR and mouse neurons is characteristic of ASIC-mediated currents [3] and to confirm the involvement of ASICs we utilized the non-selective ASIC antagonist benzamil [54]. After a first application of $\mathrm{pH} 5$ for $5 \mathrm{~s}$, we applied $100 \mu \mathrm{M}$ benzamil for $30 \mathrm{~s}$ before a second $\mathrm{pH} 5$ pulse, then followed by a wash period of $90 \mathrm{~s}$ (Fig. $2 \mathrm{~d}, \mathrm{~g})$. In cortical and hippocampal mouse neurons, the acid-induced currents were reversibly blocked by $100 \mu \mathrm{M}$ benzamil (cortex: pH 5: $133.60 \pm 19.01 \mathrm{pA} / \mathrm{pF}$; benzamil: $16.77 \pm 5.28 \mathrm{pA} / \mathrm{pF}$; wash: $133.80 \pm 19.88 \mathrm{pA} / \mathrm{pF}(n=9)$; hippocampus: $\mathrm{pH}$ 5: $151.00 \pm 33.64 \mathrm{pA} / \mathrm{pF}$; benzamil: 7.84 $\pm 2.67 \mathrm{pA} / \mathrm{pF}$; wash: $89.26 \pm 20.48 \mathrm{pA} / \mathrm{pF}(n=10)$; oneway paired ANOVA test, Tukey's multiple comparison test, ${ }^{* * * *} p<0.001,{ }^{* *} p<0.01$, Fig. 2e-f). This result concurs with previous studies indicating that acid-evoked currents in mouse hippocampal and cortical neurons are mediated by ASICs [4, 8, 15].

Similarly, in cortical and hippocampal NMR neurons, acid-induced currents were reversibly inhibited by $100 \mu \mathrm{M}$ benzamil (cortex: pH 5: $9.66 \pm 1.09$ pA/pF; benzamil: $2.78 \pm 0.49 \mathrm{pA} / \mathrm{pF}$; wash: $9.13 \pm 0.89 \mathrm{pA} / \mathrm{pF}(n=$ 7); hippocampus: $\mathrm{pH}$ 5: $11.13 \pm 1.88 \mathrm{pA} / \mathrm{pF}$; benzamil: 3 . $50 \pm 0.53 \mathrm{pA} / \mathrm{pF}$; wash: $8.15 \pm 1.30 \mathrm{pA} / \mathrm{pF}(n=6)$; oneway paired ANOVA test, Tukey's multiple comparison test, ${ }^{* * *} p<0.001,{ }^{* * *} p<0.01$, Fig. $2 \mathrm{~h}-\mathrm{i}$ ). This is the first demonstration of functional ASIC-mediated currents in CNS NMR neurons.

NMR neurons are resistant to acid-induced cell death ASICs are involved in acid-induced cell death, so-called acidotoxicity, which can occur during periods of ischemia $[5,15,16]$. Because NMR neurons exhibit significantly smaller ASIC currents (Fig. 2), we hypothesized that this could be neuroprotective when neurons are in an acidic environment. We exposed neuronal cultures from mouse and NMR cortices to a $\mathrm{pH} 7.4$ or a $\mathrm{pH} 5$ solution for $2 \mathrm{~h}$ at $37{ }^{\circ} \mathrm{C}$. Nuclei were stained by Hoechst 33,342 and necrotic (dead) neurons were labeled using propidium iodide (PI) (Fig. 3). Percentages of dead neurons were calculated by counting the number of necrotic

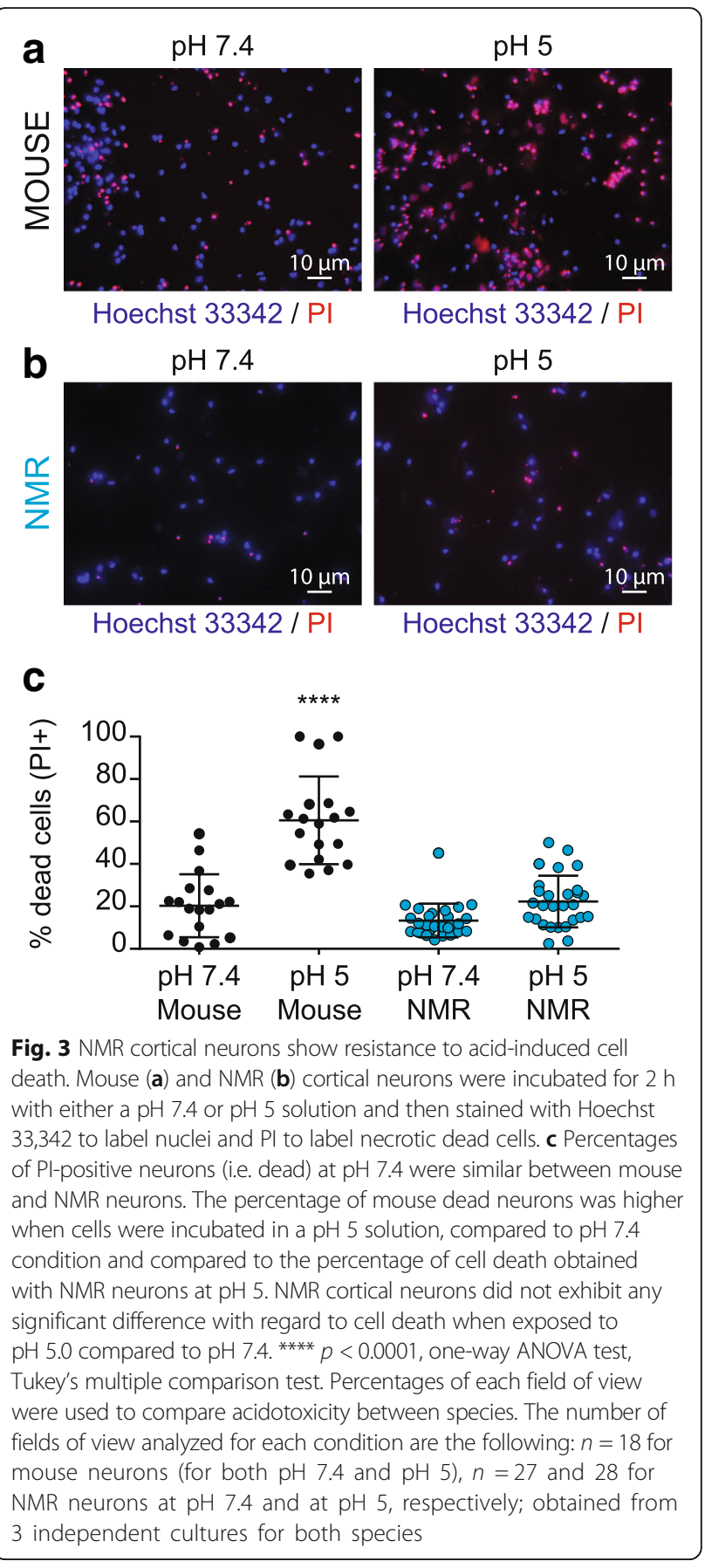

PI-positive cells over the total number of Hoechst 33,342-positive neurons in the field of view, and a oneway ANOVA test corrected for multiple comparisons (see Methods) was used to compare neuronal death at pH 5 between both species (Fig. 3c). At pH 7.4, percentages of dead cells were comparable between mouse and NMR cortical cultures (mouse - pH 7.4: $20.31 \pm 3.50 \%$, $n=18$ fields of view; NMR $-\mathrm{pH} 7.4: 13.31 \pm 1.53 \%, n=$ 27 fields of view; from 3 independent experiments), suggesting no differences in neuronal death under basal 
conditions between NMR and mouse cultures. When incubated for $2 \mathrm{~h}$ in $\mathrm{pH} 5$ solution, mouse cortical neurons exhibited a significantly increased percentage of cell death compared to all other conditions (mouse $-\mathrm{pH} 5$ : $60.55 \pm 4.87 \%, n=18$ fields of view; NMR - pH 5: 22.29 $\pm 2.30 \%, n=28$ fields of view, from 3 independent experiments, $p<0.0001$, ****). By contrast, when NMR cortical neurons were exposed to $\mathrm{pH} 5$ for $2 \mathrm{~h}$, no difference in the level of cell death was observed compared to incubation at $\mathrm{pH}$ 7.4. Similar results were obtained using mouse and NMR hippocampal neurons (data not shown). This indicates that NMR neurons are resistant to acid-induced cell death, possibly due to their reduced ASIC currents compared to mouse neurons.

\section{Discussion}

In this study, we recorded for the first time from cultured NMR brain neurons and described their basic electrophysiological properties (Fig. 1). We showed that NMR neuronal capacitance was smaller than in mouse neurons and that the resting membrane potential of NMR neurons is more hyperpolarized than in mouse neurons. One point to consider is that neurons were recorded from blindly and so we can only broadly compare hippocampal and cortical neurons between species and cannot comment on if these differences occur in all types of neuron or specific neuronal subpopulations, such as interneurons. Although capacitance and resting membrane potential values obtained from mouse neurons were similar to those reported by others using cultured rodent neurons [55, 56], for NMR neurons however, previous data from others found their resting membrane potential to be more hyperpolarized (NMR cortical neurons: $-57.03 \pm 2.64 \mathrm{mV}$, NMR hippocampal neurons: $-55.11 \pm 4.79 \mathrm{mV}$, Fig. 1; NMR hippocampal pyramidal neurons (4 months old): $-70.3 \pm 6.1 \mathrm{mV}$, NMR hippocampal dentate granule cells (4 months old): $-75.1 \pm 3.6 \mathrm{mV}$, [53]). Explanations for differences observed in resting membrane potentials between studies are the recording conditions (in vitro vs. in vivo), the developmental stage of the animal from which neurons are obtained and the constituents of the intracellular and extracellular solutions. When comparing our study with that of Penz and colleagues, who reported more hyperpolarized resting membrane potentials, two factors that could contribute to the difference observed is that we made recordings from cultured neurons, whereas they used brain slices, and secondly, we isolated neurons from neonatal animals, whereas slice recordings were made from animals aged at least 4-months.

A standard voltage-step protocol that has been already successfully used in NMR sensory neurons to predominantly isolate $\mathrm{NaV}$ activity [43] was used to measure macroscopic voltage-gated current activity in mouse and
NMR neurons. NaV currents recorded from mouse neurons were not different to what have been recorded in similar experimental conditions (for example, mouse cortical neurons at DIV 10-12: Vhalf: $-41.62 \pm 1.46 \mathrm{mV}$ in [57] versus this study: $-45.42 \pm 1.99 \mathrm{mV}$ ). Similarly, inward currents recorded in NMR brain neurons were also very similar to currents recorded in NMR sensory neurons (NMR cortex: Vhalf: $-36.12 \pm 2.80 \mathrm{mV}$; NMR hippocampus: Vhalf: $-37.10 \pm 2.34 \mathrm{mV}$; NMR DRG neurons: Vhalf: from $-34.2 \pm 0.1 \mathrm{mV}$ to $-44.6 \pm 0.5 \mathrm{mV}$ [43]). However, NMR inward currents significantly differed from mouse currents: the peak current density was significantly smaller and the Vhalf and peak $\mathrm{Vm}$ values were more depolarized. These differences in NMR NaV activity likely result from differences in amino acid sequence of $\mathrm{NaV}$ subunits and/or differential expression of accessory subunits and warrant further investigation. We also found that addition of $300 \mathrm{nM}$ TTX completely abolished all voltage-gated inward currents demonstrating an absence of TTX resistant $\mathrm{NaV}$ subunits in the NMR brain, this result aligns with the observation that the TTX-resistant NaV subunits, NaV1.5, NaV1.8 and NaV1.9 are not expressed in the central nervous system [58]. It should also be noted that although NMR neurons were cultured at $32{ }^{\circ} \mathrm{C}$ under hypoxic $\left(3 \% \mathrm{O}_{2}\right)$ conditions, recordings under normoxic, standard laboratory conditions, whereas NMRs live in a hypoxic and hypercapnic environment $[59,60]$, which may influence channel activity in vivo.

ASIC activity in brain neurons is now accepted as a key factor in numerous physiological and pathological conditions [61]. However, nothing is known about acidinduced responses in NMR brain neurons, which is of considerable interest considering the behavioral hypoxia/hypercapnia resistance and lack of acid-induced nocifensive behavior display by NMR that are likely adaptations to adapting to a safe, but relatively hypoxic and hypercapnic habitat [35]. Recently, we described ASIC subunit expression in the NMR CNS [62], which is similar to that in the mouse CNS, with the exception of lowered ASIC4 levels in the NMR brain. However, evidence for functional ASIC activity is lacking and is of particular interest in light of our recent finding that nmrASIC3 forms non-functional homomers [50]. Here, we find that NMR brain neurons produce ASICmediated currents in response to acid stimulation, in both hippocampus and cortex, as demonstrated by acid-induced responses being fully, reversibly blocked by $100 \mu \mathrm{M}$ benzamil (Fig. 2). However, the peak current density of NMR ASIC-mediated responses was significantly reduced compared to responses recorded in mouse neurons. The reasons for such reduction in ASIC currents in NMR neurons is not known and additional research is needed to determine what underpins 
this different, e.g. are regulators of ASIC plasma membrane trafficking different in NMR? Is there a different developmental expression profile of ASICs between mouse and NMR? With regard to the ASIC currents themselves, they have similar inactivation kinetics in both NMR and mouse neurons (Fig. 2c), which suggests that a similar mixture of ASIC subunits are expressed, as our previous mRNA based analysis suggested [62].

Incubation with a $\mathrm{pH} 5.0$ solution showed that unlike mouse neurons, NMR neurons do not undergo any significant acid-induced cell death (Fig. 3). This is the first demonstration of resistance to acid-induced neuronal death in NMR neurons. In rodent neurons, some factors shown to be protective against acidinduced neuronal injury, include lower temperature [63], pharmacological blockade or genetic deletion of ASIC activity [15] or ASIC trafficking [64], i.e. it is established that ASICs play a key role in acidotoxicity. Considering the similar prevalence of ASIC currents, we suggest that the reduced ASIC-mediated current amplitude observed in NMR neurons may be an additional neuroprotective mechanism in NMR brains, alongside the previously described increased hypoxiainducible transcription factor (HIF1- $\alpha$ ) expression [65] or more efficient in vivo $\mathrm{CO}_{2}$ buffering [47]. One possible mechanism for the decreased amplitude observed is reduced ASIC plasma membrane trafficking which is known to be modulated by an extracellular acidic environment [64]. However, it is also possible that the reduced acid-induced cell death observed is not ASIC-dependent because although ASIC activation appears to play a major role in neuronal injury [61], several other molecular players are also modulated by a drop in extracellular $\mathrm{pH}$, such as NaVs [27, 28] and glutamate receptors [31] and may contribute to the lowered acid-induced cell death observed, for example, here we also show that NMR neurons also have smaller NaV-mediated currents, which may also add a layer of neuroprotection.

\section{Conclusions}

In this work, we describe for the first time the basic electrophysiological properties of NMR neurons in culture and showed that the resting membrane potential of NMR neurons is more hyperpolarized, as well as the amplitude of $\mathrm{NaVs}$ being smaller than that of mouse neurons. We then demonstrated that acidinduced currents are present in NMR neurons and are, as in mouse, ASIC-mediated. The key result is that acid-induced cell death is virtually absent in NMR neurons, with reduced ASIC and NaV amplitudes likely contributing to this observation, and thus this is a further adaptation enabling NMR to live in a subterranean, hypercapnic/hypoxic environment.

\section{Abbreviations}

ASIC: Acid-sensing ion channel; CNS: Central nervous system; DRG: Dorsal root ganglion; NaV: Voltage-gated $\mathrm{Na}^{+}$channel; NMR: Naked mole-rat; TTX: Tetrodotoxin

\section{Acknowledgements}

Thanks to all members of ESS lab for their useful discussions, and in particular to the assistant staff in the Department of Pharmacology and Animal Facility for their technical help and animal husbandry.

\section{Funding}

This work was supported by an Isaac Newton Trust Research Grant from the University of Cambridge. ZH was funded by a EMBO Long-Term Fellowship (ALTF1565-2015).

\section{Availability of data and materials}

The datasets supporting the conclusions of this article are available in the University of Cambridge Apollo repository: https://doi.org/10.17863/ CAM.18704

\section{Authors' contributions}

$\mathrm{ZH}$ and ESS designed the study. ZH performed all the experiments and analysed the data. ZH and ESS wrote the manuscript. Both authors read and approved the final manuscript.

\section{Ethics approval}

All experiments were conducted in accordance with the United Kingdom Animal (Scientific Procedures) Act 1986 Amendment Regulations 2012 under a Project License (70/7705) granted to E. St. J. S. by the Home Office; the University of Cambridge Animal Welfare Ethical Review Body also approved procedures.

\section{Competing interests}

The authors declare that they have no competing interests.

\section{Publisher's Note}

Springer Nature remains neutral with regard to jurisdictional claims in published maps and institutional affiliations.

Received: 27 March 2018 Accepted: 27 April 2018

Published online: 09 May 2018

\section{References}

1. Gründer S, Pusch M. Biophysical properties of acid-sensing ion channels (ASICS). Neuropharmacology. 2015;94:9-18.

2. Waldmann R, Champigny G, Bassilana F, Heurteaux C, Lazdunski M. A proton-gated cation channel involved in acid-sensing. Nature. 1997;386: $173-7$.

3. Hesselager M, Timmermann DB, Ahring PK. pH dependency and desensitization kinetics of Heterologously expressed combinations of acidsensing Ion Channel subunits. J Biol Chem. 2004;279:11006-15.

4. Baron A, Waldmann R, Lazdunski M. ASIC-like, proton-activated currents in rat hippocampal neurons. J Physiol. 2002;539:485-94.

5. Sherwood TW, Lee KG, Gormley MG, Askwith CC. Heteromeric acid-sensing ion channels (ASICs) composed of ASIC2b and ASIC1a display Novel Channel properties and contribute to acidosis-induced neuronal death. J Neurosci. 2011:31:9723-34

6. Wu L-J, Duan B, Mei Y-D, Gao J, Chen J-G, Zhuo M, et al. Characterization of acid-sensing ion channels in dorsal horn neurons of rat spinal cord. J Biol Chem. 2004;279:43716-24

7. Gao J, Wu LJ, Xu L, Le XT. Properties of the proton-evoked currents and their modulation by CA $2+$ and $\mathrm{Zn} 2+$ in the acutely dissociated hippocampus CA1 neurons. Brain Res. 2004;1017:197-207.

8. Askwith CC, Wemmie JA, Price MP, Rokhlina T, Welsh MJ. Acid-sensing Ion Channel 2 (ASIC2) modulates ASIC1 H+-activated currents in hippocampal neurons. J Biol Chem. 2004;279:18296-305.

9. Wemmie JA, Chen J, Askwith CC, Hruska-Hageman AM, Price MP, Nolan BC, et al. The acid-activated ion channel ASIC contributes to synaptic plasticity, learning, and memory. Neuron. 2002;34:463-77. 
10. Liu MG, Li HS, Li WG, Wu YJ, Deng SN, Huang C, et al. Acid-sensing ion channel 1a contributes to hippocampal LTP inducibility through multiple mechanisms. Sci Rep. 2016:6:1-14.

11. Mango D, Braksator E, Battaglia G, Marcelli S, Mercuri NB, Feligioni M, et al. Acid-sensing ion channel 1a is required for $\mathrm{mGlu}$ receptor dependent longterm depression in the hippocampus. Pharmacol Res. 2017;119:12-9.

12. Vralsted VC, Price MP, Du J, Schnizler M, Wunsch AM, Ziemann AE, et al. Expressing acid-sensing ion channel 3 in the brain alters acid-evoked currents and impairs fear conditioning. Genes Brain Behav. 2011;10:444-50.

13. Taugher RJ, Lu Y, Fan R, Ghobbeh A, Kreple CJ, Faraci FM, et al. ASIC1A in neurons is critical for fear-related behaviors. Genes Brain Behav. 2017;16:745-55.

14. Wemmie JA, Coryell MW, Askwith CC, Lamani E, Leonard AS, Sigmund CD, et al. Overexpression of acid-sensing ion channel 1a in transgenic mice increases acquired fear-related behavior. Proc Natl Acad Sci. 2004;101:3621-6.

15. Xiong Z-G, Zhu X-M, Chu X-P, Minami M, Hey J, Wei W-L, et al. Neuroprotection in ischemia: blocking calcium-permeable acid-sensing ion channels. Cell. 2004;118:687-98.

16. Duan B, Wang $Y-Z$, Yang $T$, Chu X-P, Yu Y, Huang $Y$, et al. Extracellular Spermine exacerbates ischemic neuronal injury through sensitization of ASIC1a channels to extracellular acidosis. J Neurosci. 2011;31:2101-12.

17. Gu L, Liu X, Yang Y, Luo D, Zheng X. ASICs aggravate acidosis-induced injuries during ischemic reperfusion. Neurosci Lett. 2010;479:63-8.

18. Friese MA, Craner MJ, Etzensperger R, Vergo S, Wemmie JA, Welsh MJ, et al. Acid-sensing ion channel-1 contributes to axonal degeneration in autoimmune inflammation of the central nervous system. Nature Med. 2007;13:1483-9.

19. Chu X-P, Xiong Z-G. Physiological and pathological functions of acidsensing ion channels in the central nervous system. Curr Drug Targets. 2012;13:263-71.

20. Gonzales EB, Sumien N. Acidity and acid-sensing ion channels in the normal and Alzheimer's disease brain. J Alzheimers Dis. 2017;57:1137-44.

21. Chesler M. Regulation and modulation of pH in the brain; 2003. p. 1183-221.

22. Rehncrona S. Brain acidosis. Ann Emerg Med. 1985;14:770-6.

23. Immke DC, McCleskey EW. Lactate enhances the acid-sensing Na+ channel on ischemia-sensing neurons. Nat Neurosci. 2001;4:869-70.

24. Allen NJ, Attwell D. Modulation of ASIC channels in rat cerebellar Purkinje neurons by ischaemia-related signals. J Physiol. 2002;543:521-9.

25. Smith ES, Cadiou H, McNaughton PA. Arachidonic acid potentiates acidsensing ion channels in rat sensory neurons by a direct action. Neuroscience. 2007;145:686-98.

26. Tombaugh GC, Somjen GG. Effects of extracellular pH on voltage-gated $\mathrm{Na}$ ,$+ \mathrm{K}+$ and Ca2+ currents in isolated rat CA1 neurons. J Physiol. 1996:493: 719-32.

27. Nakamura M, Jang I-S. Acid modulation of tetrodotoxin-resistant Na+ channels in rat nociceptive neurons. Neuropharmacol. 2015:90:82-9.

28. Nakamura M, Kim DY, Jang IS. Acid modulation of tetrodotoxin-sensitive Na+ channels in large-sized trigeminal ganglion neurons. Brain Res. 2016;1651:44-52.

29. Sepulveda FV, Pablo Cid L, Teulon J, Niemeyer MI. Molecular aspects of structure, gating, and physiology of pH-sensitive background K2P and Kir K +-transport channels. Physiol Rev. 2015;95:179-217.

30. Mcdonald JW, Bhattacharyya T, Sensi SL, Lobner D, Ying HS, Canzoniero LMT, et al. Extracellular Acidity Potentiates AMPA Receptor-Mediated Cortical Neuronal Death. J Neurosci. 1998;18:6290-9.

31. Traynelis SF, Cull-Candy SG. Proton inhibition of N-methyl-D-aspartate receptors in cerebellar neurons. Lett to Nat. 1990:345:347-50.

32. Bennett NC, Faulkes CG. African mole-rats: ecology and Eusociality. Cambridge: Cambridge University Press; 2000.

33. Brett RA. The ecology of naked mole-rat colonies: burrowing, food and limiting factors. In: Sherman P, Jarvis J, Alexander R, editors. Biol. Naked Mole-Rat. Princeton: Princeton University Press; 1991. p. 137-84.

34. Jarvis JUM. Reproduction of naked mole-rats. In: Sherman P, Jarvis J, Alexander M, editors. Biol. Naked Mole-Rat. Princeton: Princeton University Press; 1991. p. 384-425.

35. Schuhmacher L, Husson Z, Smith ES. The naked mole-rat as an animal model in biomedical research: current perspectives. Open Access Anim Physiol. 2015;7:137.

36. Ruby JG, Smith M, Buffenstein R. Naked mole-rat mortality rates defy gompertzian laws by not increasing with age. Elife. 2018;7:1-18.

37. Edrey YH, Medina DX, Gaczynska M, Osmulski PA, Oddo S, Caccamo A, et al. Amyloid beta and the longest-lived rodent: the naked mole-rat as a model for natural protection from alzheimer's disease. Neurobiol Aging. 2013;34:2352-60.
38. Orr ME, Garbarino VR, Salinas A, Buffenstein R. Sustained high levels of neuroprotective, high molecular weight, phosphorylated tau in the longestlived rodent. Neurobiol Aging. 2015;36:1496-504.

39. Seluanov A, Hine C, Azpurua J, Feigenson M, Bozzella M, Mao Z, et al. Hypersensitivity to contact inhibition provides a clue to cancer resistance of naked mole-rat. Proc Natl Acad Sci U S A. 2009;106:19352-7.

40. Tian X, Azpurua J, Hine C, Vaidya A, Myakishev-Rempel M, Ablaeva J, et al. High-molecular-mass hyaluronan mediates the cancer resistance of the naked mole rat. Nature. 2013:499:346-9.

41. Liang S, Mele J, Wu Y, Buffenstein R, Hornsby PJ. Resistance to experimental tumorigenesis in cells of a long-lived mammal, the naked mole-rat (Heterocephalus glaber). Aging Cell. 2010;9:626-35.

42. Park TJ, Lu Y, Jüttner R, Smith ESJ, Hu J, Brand A, et al. Selective inflammatory pain insensitivity in the African naked mole-rat (Heterocephalus glaber). PLoS Biol. 2008;6:0156-70.

43. Smith ESJ, Omerbašić D, Lechner SG, Anirudhan G, Lapatsina L, Lewin GR. The molecular basis of acid insensitivity in the African naked mole-rat. Science. 2011;334:1557-60.

44. Smith ESJ, Blass GRC, Lewin GR, Park TJ. Absence of histamine-induced itch in the African naked mole-rat and "rescue" by substance P. Mol Pain. 2010;6:29.

45. Lavinka PC, Brand A, Landau VJ, Wirtshafter D, Park TJ. Extreme tolerance to ammonia fumes in African naked mole-rats: animals that naturally lack neuropeptides from trigeminal chemosensory nerve fibers. J Comp Physiol A Neuroethol Sensory Neural Behav Physiol. 2009;195:419-27.

46. Larson J, Park TJ. Extreme hypoxia tolerance of naked mole-rat brain. Neuroreport. 2009;20:1634-7.

47. Park TJ, Reznick J, Peterson BL, Blass G, Omerba D, Bennett NC, et al. Fructose-driven glycolysis supports anoxia resistance in the naked mole-rat. Science. 2017;311:307-11.

48. Peterson BL, Larson J, Buffenstein R, Park TJ, Fall CP. Blunted neuronal calcium response to hypoxia in naked mole-rat hippocampus. PLoS One. 2012;7:1-8.

49. Peterson BL, Park TJ, Larson J. Adult naked mole-rat brain retains the NMDA receptor subunit GluN2D associated with hypoxia tolerance in neonatal mammals. Neurosci Lett. 2012;506:342-5.

50. Schuhmacher L-N, Callejo G, Srivats S, Smith ESJ. Naked mole-rat acidsensing ion channel 3 forms nonfunctional homomers, but functional heteromers. J Biol Chem. 2018;293:1756-66.

51. Schuhmacher L, Smith ESJ. Expression of acid-sensing ion channels and selection of reference genes in mouse and naked mole rat. Mol Brain. 2016; 9(1):97.

52. Omerbašić D, Smith ESJ, Moroni M, Homfeld J, Eigenbrod O, Bennett NC, et al. Hypofunctional TrkA accounts for the absence of pain sensitization in the African naked mole-rat. Cell Rep. 2016;17:748-58.

53. Penz OK, Fuzik J, Kurek AB, Romanov R, Larson J, Park TJ, et al. Protracted brain development in a rodent model of extreme longevity. Sci Rep. 2015:5:11592.

54. Baron A, Lingueglia E. Pharmacology of acid-sensing ion channels physiological and therapeutical perspectives. Neuropharmacology. 2015;94: 19-35.

55. Evans MS, Collings MA, Brewer GJ. Electrophysiology of embryonic, adult and aged rat hippocampal neurons in serum-free culture. J Neurosci Methods. 1998:79:37-46.

56. Yang J, Thio LL, Clifford DB, Zorumski CF. Electrophysiological properties of identified postnatal rat hippocampal pyramidal neurons in primary culture. Dev Brain Res. 1993;71:19-26.

57. Wang X, Zhang XG, Zhou TT, Li N, Jang CY, Xiao ZC, et al. Elevated neuronal excitability due to modulation of the voltage-gated sodium channel Nav1.6 by AB1-42. Front Neurosci. 2016;10:1-9.

58. Catterall WA. Voltage-gated sodium channels at 60 : structure, function and. Pathophysiology. 2012;11:2577-89.

59. Shams I, Avivi A, Nevo E. Oxygen and carbon dioxide fluctuations in burrows of subterranean blind mole rats indicate tolerance to hypoxic - hypercapnic stresses. Comp Biochem Physiol A Mol Integr Physiol. 2005;142:376-82.

60. Mcnab BK. The metabolism of fossorial rodents: a study of convergence. Ecol Soc Am. 1966:47:712-33.

61. Huang Y, Jiang N, Li J, Ji Y-H, Xiong Z-G, Zha X. Two aspects of ASIC function: synaptic plasticity and neuronal injury. Neuropharmacology. 2015;94:1-6.

62. Schuhmacher L, St E, Smith J. Expression of acid-sensing ion channels and selection of reference genes in mouse and naked mole rat. Mol Brain. 2016: $1-12$. 
63. Ding D, Moskowitz SI, Li R, Lee SB, Esteban M, Tomaselli K, et al. Acidosis induces necrosis and apoptosis of cultured hippocampal neurons. Exp Neurol. 2000;162:1-12.

64. Zeng $W-Z$, Liu D-S, Buan B, Song X-L, Wang X, Wei D, et al. Molecular mechanism of constitutive endocytosis of acid-sensing Ion Channel 1a and its protective function in acidosis-induced neuronal death. J Neurosci. 2013;33:7066-78

65. Xiao B, Wang S, Yang G, Sun X, Zhao S, Lin L, et al. HIF-1a contributes to hypoxia adaptation of the naked mole rat. Oncotarget. 2017:8:109941-51

Ready to submit your research? Choose BMC and benefit from:

- fast, convenient online submission

- thorough peer review by experienced researchers in your field

- rapid publication on acceptance

- support for research data, including large and complex data types

- gold Open Access which fosters wider collaboration and increased citations

- maximum visibility for your research: over $100 \mathrm{M}$ website views per year 